\title{
Eye Space: An Analytical Framework for the Screen-Mediated Relationship in Video Games
}

\author{
Yu-Ching Chang, Chi-Min Hsieh \\ Institute of Applied Arts, National Chiao Tung University, Taiwan \\ Email: rainnysunny@gmail.com
}

How to cite this paper: Chang, Y.-C., \& Hsieh, C.-M. (2017). Eye Space: An Analytical Framework for the Screen-Mediated Relationship in Video Games. Art and Design Review, 5, 84-101.

https://doi.org/10.4236/adr.2017.51007

Received: January 14, 2017

Accepted: February 25, 2017

Published: February 28, 2017

Copyright $\odot 2017$ by authors and Scientific Research Publishing Inc. This work is licensed under the Creative Commons Attribution International License (CC BY 4.0).

http://creativecommons.org/licenses/by/4.0/

(c) (i) Open Access

\begin{abstract}
This article explores the connections between players and game worlds through the screen-mediated space in games-i.e., eye space. Eye space is the crucial link between players and the game world; it's the decisive area where the gameplay takes place. However, the concept of eye space is not attention to and is frequently confused with game space because the two are closely connected and sometimes they can even interchange with each other. Thus, the study focuses on the basic building blocks and the structure of eye space in games. An analytical framework based on the existing literature and practice is proposed with special attention on the interactive nature of video games in order to examine the interplay between players and game spaces. The framework encompasses three aspects: the visual elements within the eye space, the relation of eye space to the players, and the interaction of eye space with the game space. This study aims to establish a common language to describe the screen-mediated space in games and provide further insight into game design.
\end{abstract}

\section{Keywords}

Video Games, Eye Space, Gameplay, Game Design, Game Space

\section{Introduction}

"Video game worlds are navigable spaces that offer a full range of interactions, but they are also spaces told to us using certain forms of presentation." (Nitsche, 2008) In other words, video game worlds are confined to a screen space, monitor, or even augmented reality (AR) glasses; through the screen-mediated spaces, they begin to emerge and communicate with players. Therefore, certain cameras have to be set in game spaces, and certain selections have to be made by game designers. The complexity, richness, and quality of the virtual game world-infused with a viewpoint through design and framing-build connections between players and the game world. Generally, the viewpoint comes from a virtual cam- 
era, or, as Manovich (2001) states, "an arbitrary point of view-a picture of a virtual world recorded by a virtual camera." Despite early or two-dimensional (2D) games, it is not necessary to use any kind of virtual camera or even have the concept of a camera. Game designers still need to scheme all gameplay components for the presentation of the visual screen in order to communicate with players. When eye space has been sketched out, players can experience its perception, and the connections between players and game worlds can be built. This is an indispensable process of game design as well as an underdeveloped research area.

The term "eye space" is employed in this article for the more active participatory nature of games as well as to distinguish from the cinematic "on- and offscreen space" as well as "camera space", which generally indicates the virtual camera systems in games. The notion of eye space has often been mentioned in discussions related to game space and user interface, but it has rarely been looked at closely, nor does eye space have a common language and understanding. Game space is manifold, from basic rule-based systems to fictional narratives and all the way to the real social sphere. Wolf (2001) has examined screenbased game space and set different representations and particularities into relation. "However, in an attempt to formulate a spatial taxonomy, Wolf mixes qualities of game spaces such as depth of space and point of view or traversability/navigation and representation of space." (Walz, 2010) That is, the concepts of eye space and game space are frequently confused with each other because they are tightly connected. Eye space is the graphical entrance for game players. In a broad sense, eye space is a part of user interface, which is the central component of any video game, and "mediates between the core mechanics of the game and the player" (Adams, 2009). Nitsche (2008) identifies five analytical planes for the analysis of game space: rule-based space, mediated space, fictional space, play space and social space. Eye space mostly resembles mediated space, which is defined by the presentation; nonetheless, at the same time, it stretches out to rule-based and play spaces, which are not merely visual. In other words, eye space defines not only the appearance of game space but also the ways in which players engage during gameplay. As Wood (2012) stated: "While cameras do not affect the action, when associated with the moving viewpoint of an avatar, the camera defines both the space of the game and the way in which the viewer is embedded in that game. The camera is more than an element through which a gamer enters into the game world, it mediates as the input of the gamer is translated into the reconfigurations of space."

To this end, the theoretical concern of this research is to delve into eye space as the vital linkage between players and game spaces as shown in Figure 1. The analytical perspective of screen-mediated eye space is addressed in three aspects. First, we extend the concept of photographic composition as the building blocks of design elements inside eye space and discuss their functionalities, visual purposes and effects on gameplay. Second, eye space must be derived from a distinct standpoint that significantly affects the degree of identification and gameplay 


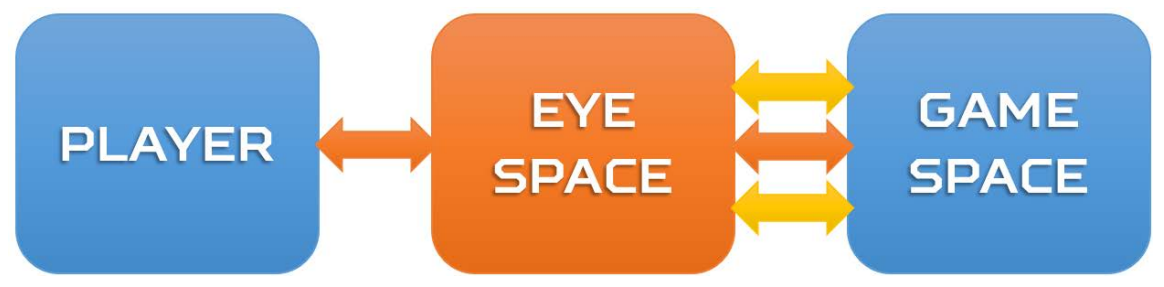

Figure 1. The analytical framework of Eye Space.

experience of players. Flynn (2004) argues that the discussions of spatiality in video games need to be taken into account, for "the participatory and embodied positions of the player". Therefore, it is necessary to describe the different presentations of eye space regarding the players' locus of manipulation with more detail. Last, we address the interplay between eye space and game space-in terms of eye space as the interface of the game world-that not only open up players' participation, communication and experience, but also sculpt the appearance of game space. The research focuses on these three aspects within the scope of game design in order to establish a common language for eye space and attempts to construct an analytical framework.

\section{Inside Eye Space}

As an entertainment medium, a video game, "although rich with unique potential of its own, shares elements in common with other arts", (Rollings \& Morris, 2003) namely, the idea of composition in visual communication design, which is the deliberate manipulation and arrangement of lines, shapes, colors, focus, tones, etc., in a work of art. Based on these visual ingredients, films add the element of time and use cameras to create unique visual grammar, the "invisible technique" (Ward, 2002), or the concept of mise-en-scène. According to Nitsche (2005), "framing, mise-en-scène and montage are part of the video game world". Additionally, Logas and Muller (2005) contend that "examining the ways miseen-scène is used in films will help designers adapt a holistic approach to their own discipline and create deeper resonance with the player", except their main topic is about the effects of space composition on emotion and atmosphere in the horror genre.

In the meantime, scholars have pointed out that "digital games are fundamentally different to 'traditional' media forms due to their 'interactive' nature" (Gosling \& Crawford, 2011). Because of their participatory nature, games become unique for offering players a rule-based combination of challenge, exploration, and reward mechanisms while being limited by the capacity of the screen, which is similar to the camera frames in films, but one has to allow more consideration for the practical purposes of gameplay. As Nitsche (2008) stated, "functionality refers to the interactive access and underlying rules determining what the player can do in the game space and what space can do to adjust that". Visual presentation in a game should always take the functional intent into account; "any concentration on either presentation or functionality but not both would destroy the holistic principle of spatial experience". 
To this degree, while referencing the cinematography principles of visual composition, the placement of foreground, background, and mise-en-scène, functional design also brings eye space configuration into analysis. Four basic design components have been defined in this manner. The following discussion is devoted chiefly to the four specific design components of eye space and will be explained individually in terms of its representation, purpose, and mutual relations with players.

A. Primary Subject:

In the field of painting and photography, centers of interest are the theme of the image, the core of the composition, and the most interesting part for viewers. In eye space games, the primary subject is akin to the center of interest. It usually has noticeable features to grab players' eyes and attention and also provides necessary information for gameplay. Diverse primary subjects are used for different purposes within different game genres and goals, but they share the same goal: to maintain the flow of a game. In other words, most of the primary subjects can affect the winning and losing of a game. Games also rely on the concentration of players and directly reflect their input and manipulation on action controls. For example, Mario is the player-controlled character in Super Mario Bros. (1985) who constantly sticks around the middle of the screen as the prominent part of the image, whereas Pac-Man (1980) is also a player-controlled character-with bright yellow corresponding to the dark background-who rambles all over the place and collects points while avoiding ghosts. As these two examples imply, elements such as position and color can emphasize the primary subject within the frame, while immediate control and manipulation-the most familiar linkages between players and games-are the critical factors when deciding who the main subject is. There are exceptional cases, though, like the one in Pinball (1980), where players pay much more attention to the ball rather than the player-controlled flippers. It is worth it to mention that control and manipulation still have the most significance on the primary subject because playercontrolled flippers impact the rebound angles and movements of the ball.

Mobility and player alternatives are also features of the primary subject, especially when there are more than one controllable objects, or potential primary subjects. Tetris (1984) is the obvious example, as players have to manipulate the randomly popping up tetriminos one by one with the aim of creating a horizontal line without gaps; every tetrimino becomes the primary subject for a time (from the time it appears until it reaches the ground). As another example, in Warcraft (1994), players are busy constructing architectures for defense while gathering resources, developing technologies, and commanding orders for troops. As shown in Figure 2, each time the player makes a selection, the unit currently selected is marked with a light green box, which immediately becomes the primary subject, and its details appear in the status panel; until the player selects a different unit and the primary subject shifts. A bunch of armies might be the selected unit; this shows that the primary subject could be multiple, or more than one object. As mentioned previously, different game genres and goals have an influence on the quality of the primary subject. 


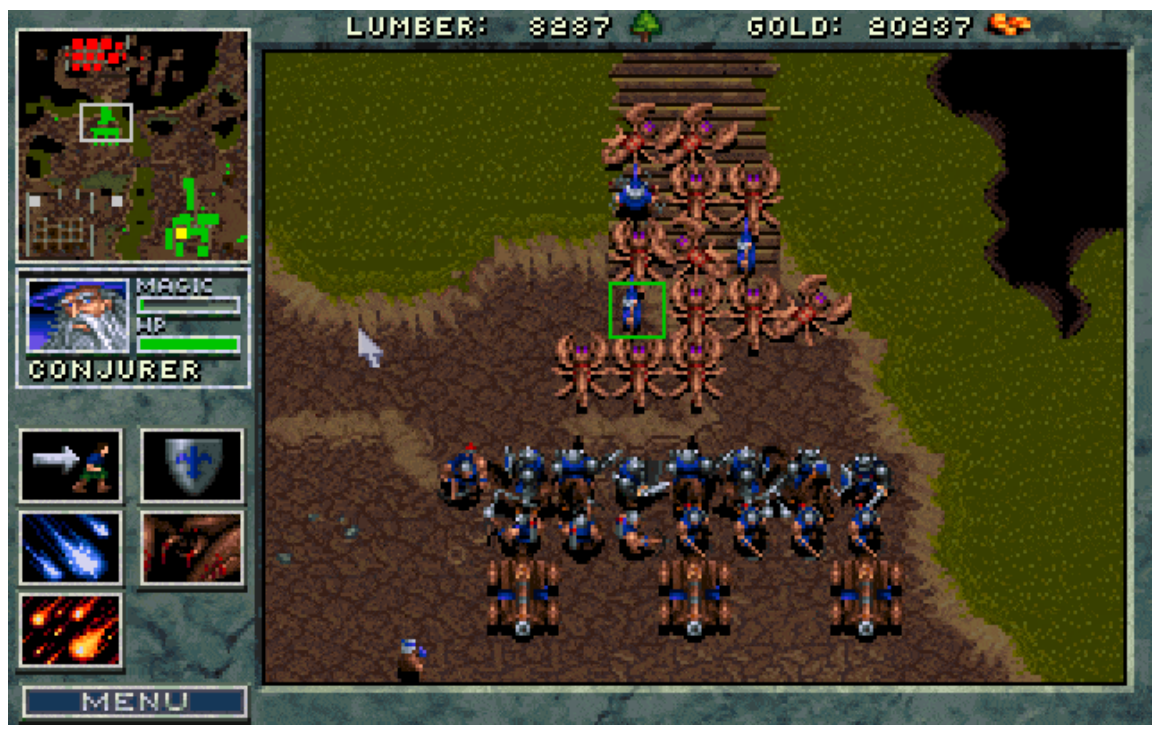

Figure 2. Screenshot from Warcraft (Blizzard, 1994).

A picture without a dominant center of interest, or one with more than one, is puzzling to a viewer; namely, a conspicuous theme is essential for painting and photography that clearly conveys the context and meaning. Nevertheless, it is creative and rule breaking, which is what a game design element can do. Some games deliberately bury the primary subject or place it somewhere that players have to try hard to find. When there is nothing particular on the screen to draw attention to it, the eyes wander throughout the screen space. Hidden object games use this strategy to challenge players to search screen after screen for the assigned items. First-person shooter games, at the other extreme, give players full power to decide where to perceive and which part to lay eyes on. In this situation, eye space itself becomes the primary subject and gives players a different way to interact and experience the game space in a way that also increases the difficulty of gameplay.

B. Distractions

To a large extent, distractions-the secondary subjects-are what players ought to pay attention to most of the time, except for the primary subject; this is a key factor for maintaining gameplay. Distractions can be various kinds of things, including barriers, enemies, exits, event trigger objects, or anything that matters to players and makes games more abundant in many aspect, such as providing challenges, selections, rewards, and fulfillment for players. Every now and then distractions might convert into primary subjects for a while when they occupy more space or players need to focus better. A boss battle is the classic reverse case. For instance, in the normal gameplay of the Raiden series, players control the fighter-who is the primary subject-to avoid bullets, get power-ups, collect medals, and shoot every enemy fly-in from everywhere on the screen. All of these things are distractions. However, when the boss battle begins, as shown in Figure 3, the scrolling screen now stops and presents as a duel scene, and the boss, who is usually huge compared to other enemies, becomes a primary subject along with the player-controlled fighter. 


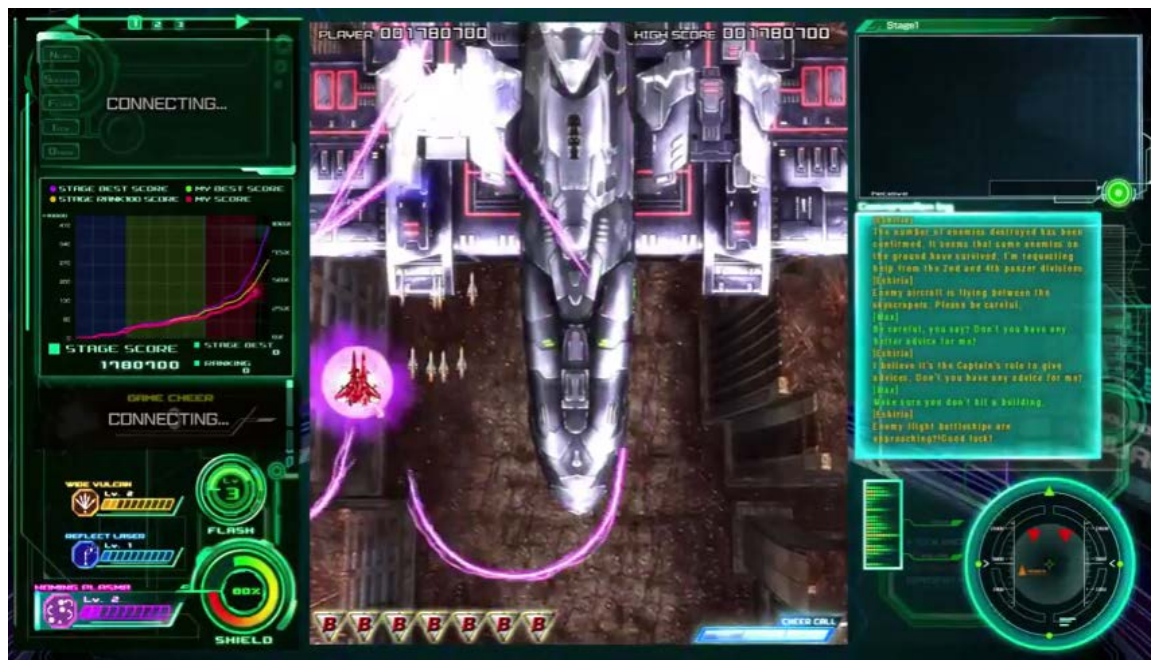

Figure 3. One of the boss mission in Raiden V (MOSS, 2016).

It is not necessary to directly link distractions, player control, and manipulation. In general, players cannot directly control distractions. In terms of game mechanics, distractions are not indispensable; they play different roles in different games and genres. While players may sometimes devote all their attention to distractions, others may dismiss them as trivial decorations. Yet, distractions have a degree of effect and interference in gameplay. For players, these things are meaningful, functional, and necessary for their immersion experience. In addition, the design and arrangement of distractions has to consider affordances, which "refers to the perceived and actual properties of the thing, primarily those fundamental properties that determine just how the thing could possibly be used" (Norman, 2002). Affordances may suggest strong clues with their appearances or other attributes to their operations. "An artifact's perceived affordances have the ability to improve players' progression through the game" (Bacher, 2008); that is, in the right context and with proper indication, distractions can be critical linkages that connect players with immersive gameplay.

C. Backdrop

Assuming that primary subjects and distractions are in the foreground (although they are not necessarily situated in the front of a scene), the backdrop constructs the background and environmental details, although it is not necessarily situated in the back. While backdrop seems to have nothing to do with gameplay other than merely acting as visual decoration, it in fact has a lot to do with it. The backdrop fills out the blanks and increases the authenticity of eye space so the primary subjects and distractions can be more persuasive. "The primary purpose of architecture is to control a person's experience." (Schell, 2008) This provides a consistent experience for the player as well as the ambiance of the game, which is "everything that contributes to the innate look and feel of the game" (Rollings \& Morris, 2003). Take Silent Hill (1999) for example. Without the dark and narrow corridors, rusty and blood-stained walls, and disturbing and nasty bathrooms, it would not be successful because it would lack 
the horror and suspense atmosphere. The backdrop can be perfectly helpful when establishing the game theme and storyline.

The abundant visual details of the backdrop create the ambiance and atmosphere of eye space and also provide a context for players to better immerse themselves in games, even though players do not have any power to change or intervene in the backdrop. On the contrary, the backdrop could have a say in gameplay due to the nature of the game design. Everything can be a part of an interactive trigger, from simple lines, such as the ones in Snake games that define the boundaries (Figure 4), to more complicated objects like stairs, trees, buildings, and water surfaces, which have their own functionalities aside from their appearances. Objects in games generally have one or more attributes, which are "categories of information about an object" (Schell, 2008). Take Excitebike (1984) for instance. The backdrop use distinct colors to separate audience bleachers, racing tracks, and the speed-lowering grass (Figure 5). Therefore, not only does the backdrop define the main space for gameplay, but it also affects gameplay on different aspects.

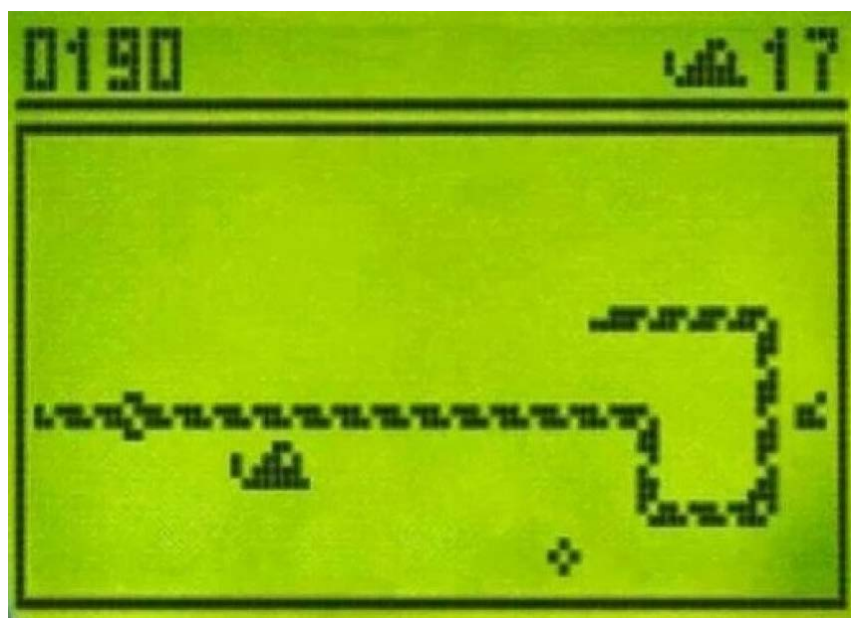

Figure 4. Snake (Nokia, 1998).

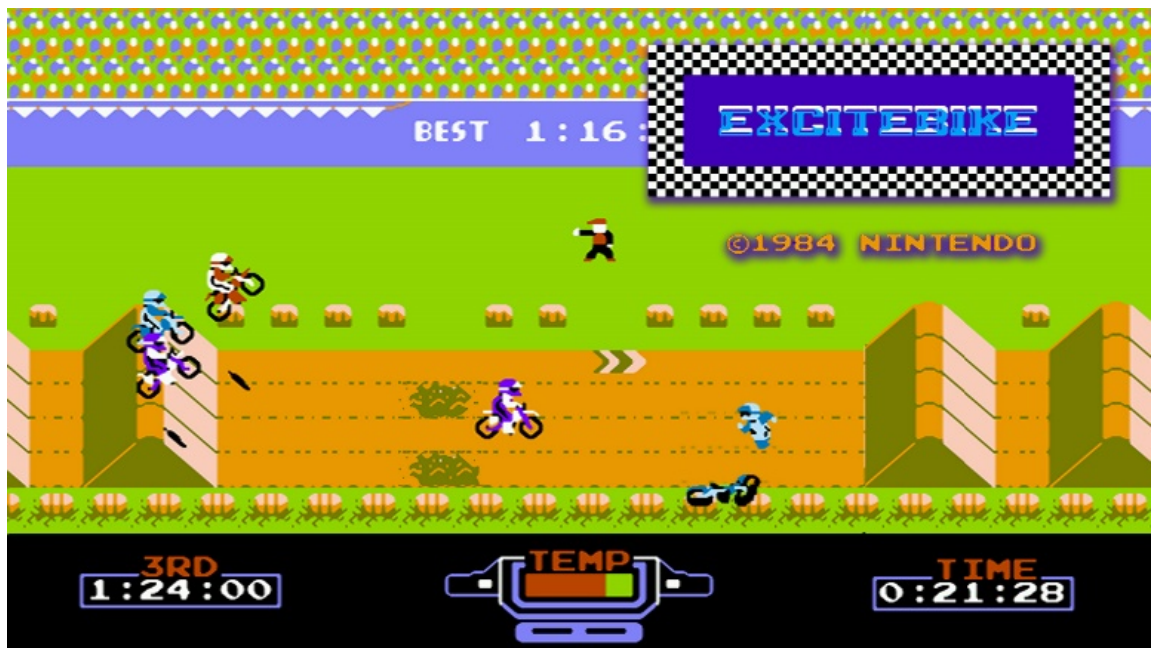

Figure 5. Excitebike (Nintendo, 1984). 


\section{Guiding information}

Guiding information is the visual instructions and cues in eye space distributed to players; for example, the light green box in Warcraft, that marks the selected unit, the judgments in Dance Dance Revolution (1998) that rate players' timing when hitting the arrows, and the predicted trajectory path in the dotted lines in Angry Birds (2009). Generally speaking, it is part of the graphical user interface (GUI) and clear evidence of data visualization in games (Bowman, Elmqvist, \& Jankun-Kelly, 2012), such as the status bar and the heads-up display commonly used in point of view games. This guiding information can be long term or short term depending on its type and purpose. Most of the heads-up displays are static onscreen, so they stay visible during gameplay. However, immediate messages and responses can give practical support to players most of the time. Besides, it is crucial to use the right technique to design the data that will be noticed but not interfere with the players. Fagerholt and Lorentzon (2009) introduced six categories for different types of user interface elements depending on how they linked to the narrative (diegesis) and game geometry (spatiality). These various on-screen displays are like layers on the game screen; they do not only inform players of their current progress and achievements, but also help create a more immersive game space.

These components are not fixed, but may vary and transition with each other in relation to the progress of gameplay. In addition, the components inside eye space define the basic architecture of the screen-mediated space and make sure the core mechanics are well designed and presented in the eye space; at the same time, they bridge the real and virtual worlds. Through eye space, players are able to communicate with the game space. However, there are two segments that can be further discussed that relate to the process that raises issues Behind eye space and beyond eye space, which implies eye space in relation to players as well as to game space.

\section{Behind Eye Space: Players}

Viewpoint derives from a particular line of sight and describes the position of the subject (the avatar) to the viewer, the player, and the vanishing point of space in games, merging "spectatorship and participation in ways that fundamentally change both activities" (Rehak, 2003). When serving as a narrative technique, viewpoint can be defined as the angle from which the story is seen and recounted; that is, the point of view of the narrator, such as first person, third-person limited and third-person omniscient. However, the game narrator and player are not necessarily the same person, nor do all games have stories. Therefore, traditional literary narrative forms need to have some modifications in order to answer the particular needs in games. Nitsche (2008) has discussed three types of player positioning in games; he argued, "players ['you'] are not directly projected into the fictional world of a video game space. Instead, they [you] get access to distinct elements (e.g., an avatar) within it and from that, a feeling of presence can emerge". That is, the eye space to which players are 
guided in order to immerse themselves in games with their consciousness and identities will not be deprived but rather transferred into the game; it will become a distinct standpoint regarding the feature of the gameplay and background stories for which players may become engaged. Based on the above analyses, our research emphasis is placed on the locus of manipulation as well as visual representation. Symbolization of self is the fundamental purpose of viewpoint in games, as well as the representation of players and what they can take control of during gameplay. In other words, this section analyzes player participation regarding eye space at two levels as shown in Figure 6: the locus of manipulation and the ways of presentation.

Sherry Turkle pointed out that when a player plays a game, "you have to do more than identify with a character on the screen. You must act for it." (Turkle, 2005, first published in 1984) Likewise, Meldgaard (2008) holds that "the understanding of the role of perception in game space, must be viewed in correlation with the actions made possible". Thus, the notion of locus of manipulation arose, a description of "the in-game position of the player's ability to assert control over the game world" (Lankes, Mirlacher, Wagner, \& Hochleitner, 2014). From this standpoint, many articles study player identification, functional representation, and game immersion in various degrees (Bayliss, 2007; Gazzard, 2013; Lankes et al., 2014; Linderoth, 2005). In contrast, eye space examines ways to present viewpoint perspectives and focuses on design metaphors; game space projects a certain controlling perspective that players take on in games with a dynamic nature of engagement and embodiment. Among the various manifestations of interactive modes for players, there are four possible solutions for design metaphors: protagonist, commander, invisible man, and observer.

The protagonist metaphor is one-to-one. A player takes on the role of one certain character at a time; the actions the player makes and its inputs are directly channeled through this particular character, such as Sonic in Sonic the Hedgehog (1991). A similar concept is the common trope of player-character, or when "players experience games through the exclusive intermediary of another-the avatar-the 'eyes', 'ears', and 'body' of which are components of a complex technological and psychological apparatus" (Rehak, 2003). Under this circumstance, the objective of eye space is to convey everything players need to

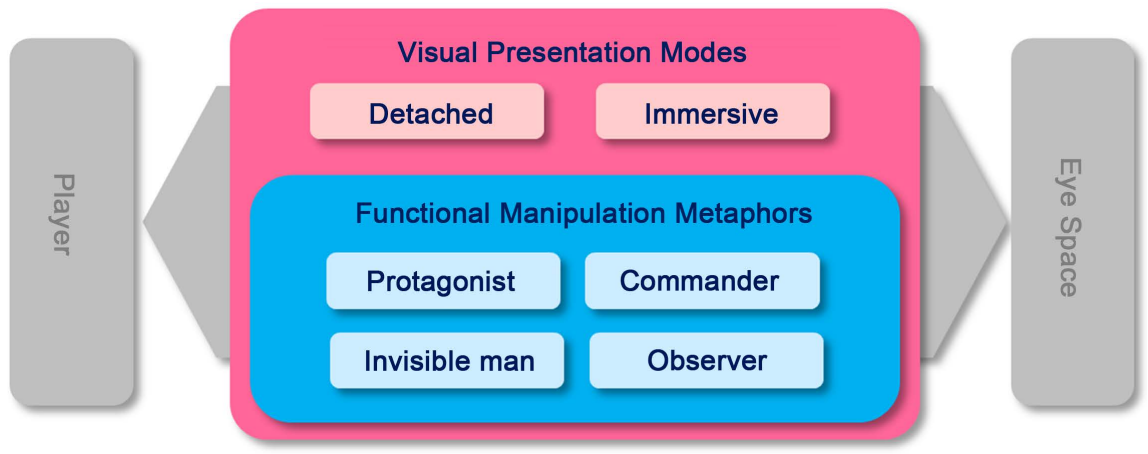

Figure 6. Two levels of the player participation structure behind eye space. 
know about the role and character of which they are in control, including the actions they make, circumstances surrounded, basic status of the character, and even the character progression tree. Players tend to be more inclined toward the character they control; however, players do not necessarily identify themselves with the character or role they are assigned when considering the psychological variables and varying natures of the viewpoint. The distinct linkage can be made by a certain locus of manipulation that was precisely schemed by game designers. Humans or mechanics, living or nonliving things-the connections based on actual entities with intuitive operations and narratives often play a supportive role.

The commander metaphor is one-to-many; players take control of multiple entities. When preparing for the role the player must take on, the standpoint evolves into a higher level, and multitasking conditions ensue. For example, in Starcraft (1998), players serve as the ruler of a distinct race whose goal is to survive and conquer its enemies; thus, they have to train armies to fight against others and protect the terrain while upgrading their defense and developing technologies. Players cannot be confined by the concern of performing the right moves for a single character with the controller; they have to have a broad and comprehensive sight instead. This relates to the notion of God view: "Gameplay in these games is based on the concept that the player is a force that acts upon the world of the game, rather than a force within the game that then acts on the objects and actors of the game from within" (Taylor, 2002). The difference is that commander mode has a more clear and definite existence and authority; it prevails in construction and management simulation (CMS) (Adams, 2009) and eXplore, eXpand, eXploit, and eXterminate (4X) games (Emrich, 1993). The exertion of player control is not direct but more indirect because the target subjects are a number of individuals. As a result, instructs and orders need to be transformed and pointed to specific spheres or groups. Accordingly, these games always incorporate numerous and diverse visual representations in eye space, which can deliver a more immersive experience to the players.

The invisible man metaphor does not have a particular corresponding state. It is a rather vague and ambiguous force, such as the invisible hand in Lemmings (1991). Its common form of player-character is the cursor, "the minimal form of third-person avatars" (Klevjer, 2012). Players may not know what role they are taking on or have, any clues as to the context-it is an implied role as the Supreme Being. In some games, there is even "no avatar in the game space. Instead, the player represents him- or herself" (Brathwaite \& Schreiber, 2008). Compared to the former modes, the lack of a clear identification has a more familiar and intimate manner of viewing. Moreover, the core game mechanics can be revealed more directly to players. In Tetris, for example, there are no clues regarding the positions of the players, only controllable blocks in space that plainly point out the methods designed for interaction in which players can be as engaged as other games. The invisible manners also leave greater room for players to have imaginations and develop feelings. 
The observer metaphor is different from the formers; players mainly do not have actual control, but just watch. In this case, players have minimal gameplay; for example, interactive fiction games, cut scenes, and scoring screens. The interactions between players and games are mostly one-way or straight to the players as receivers. For instance, in Heavy Rain (2010) and The Walking Dead (2012), which are interactive fiction games, the gameplay is more like reading a graphic novel or watching a story unfold in front of players. The interactions consist of clicking and making decisions to keep the story moving forward.

These different metaphors of eye space coupled with the narratives construct the multiple identities for players that result in the unique charm of human-computer interaction in games; the connections between the two are inseparable. An equally important issue is how to describe these metaphors. The traditional first- and third-person point of views have now become ambiguous; popular games such as World of Warcraft (2004), Minecraft (2010), or Grand Theft Auto V (2013) all support players to willingly change between the two perspectives and even the viewing distance. As Thon (2009) noted, "many contemporary computer games allow their players an ever greater amount of control over the spatial perspective(s) used in the presentation of the game space." As a result, presentation of the viewpoint should roughly be redefined into two modes:

Immersive mode: Eye space creates a surrounding virtual environment where the player is immersed in the act of the gameplay experience. This can often be found in shooter games. Despite the first-person point of view that aligns to match the viewpoints between the character and the player, or the third-person point of view where the character can be seen in the viewpoint, the actions that players make can promptly respond onscreen with a great deal of virtual reality and visual impact. Apart from that, predefined narrative viewpoints, such as the one in Heavy Rain-which has many movie shooting and editing techniques, particularly cinematic montage-result in increased tension along with the storyline and audiovisual spectacle. Players can be visually enriched with a great and powerful sense of immersive gaming experiences.

Detached mode: This is a quite objective and plain view. Players take a relatively distant position to the subject and game space, so they can barely experience the impacts from the visual environment. However, more and better information is available to them. Game series like Civilization and Age of Empires are always presented this way in order to show broad fields and a lot of objects. On the other hand, for certain gameplay mechanics, keeping the visual fields as simple as possible is needed in order for players to focus on more important things; for example, the moves and actions in the Street Fighter series, or the obstacles and enemies in side-scrolling games such as Super Mario Bros.

\section{Beyond Eye Space: Game Space}

Eye space is a small part of game space that peeps into the game and shows a specific area of the whole game world. This is typically the case in most games, 
apart from the earliest games and some specific game types such as puzzle games, in which the complete game space is condensed onto one single screen. In fact, eye space has become diverse with the ever-increasing technology innovations and complexities of game spaces. The most related topic is the term of the virtual camera in three-dimensional (3D) games, which is exactly the concrete representation of eye space. There are many studies on the technical improvements and automations of virtual camera systems concerning the convenience and accuracy of viewpoint manipulation in games, or bringing in the skills of cinematography from film productions for a more expressive composition (Burelli, 2013; Kneafsey, 2006; Lin, Shih, \& Tsai, 2004). However, it is not always necessary for games to have a camera, especially in $2 \mathrm{D}$ games. Some of them use the techniques in 3D game engines, such as the camera projection in Unity that renders an isometric view. Other games apply multilayer parallax scrolling effects to create a sense of depth; for instance, ActRaiser (1990), Shovel Knight (2014), or Pole Position (1982), which is said to be the first realistic racing game with $3 \mathrm{D}$ graphics. In fact, the simple scaling road sprites give the illusion of $3 \mathrm{D}$ effects. Despite the approaches in 2D or 3D games, it can be said that there is a very clear difference between eye space and game space. Game space itself remains unchanged most of the time; what changes is the way it is framed in the eye space. Therefore, the way these two communicate is delicate and has a strong impact on player gameplay.

Generally speaking, in contrast to constant game space, eye space is fluid and reveals the game in a mobile way. As game mechanics grow various and game space becomes complicated, a single game space could contain one or more different forms of eye space but not be limited to one particular type. Besides, the varied forms can operate with each other in different situations to create a more flexible and immersive world. The focus here is on the underlying connections between game space and eye space, which has been divided into two aspects as shown in Figure 7: visual and interactive.

First of all, the visual relations between game space and eye space are primarily determined by the viewing angles and viewpoint fields, which can refer to the

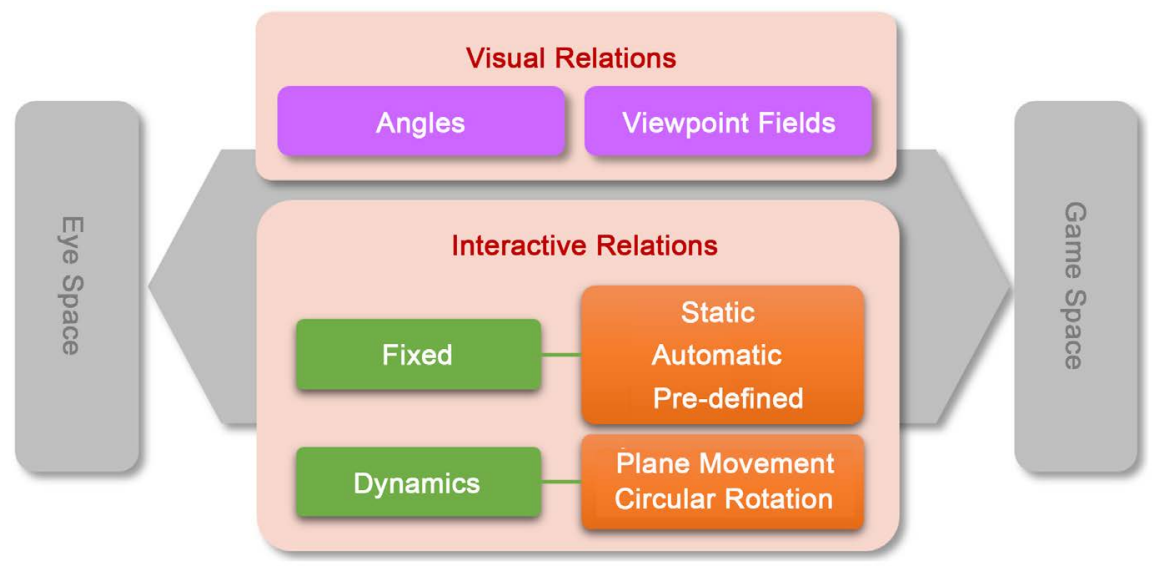

Figure 7. The structural relations beyond eye space. 
concept of camera language in cinema. Different viewing angles can affect players' perceptions of space, perspectives, and senses. Choosing the most advantageous angle to show the important details is of great importance and is closely related to the game's genre. Normally, viewing angles can be divided into three types: high, eye level, and low angles. High angles are usually associated with the top-down genre of many strategy games, simulation games, and some roleplaying games like Diablo (1996) and the Final Fantasy series. Looking down from a high angle can make the scene more dramatic and also give players a wider view; therefore, it can deliver more information on the surroundings in gameplay so players can make timely responses. High-angle shots, as a cinematic technique, tend to emphasize the smallness or insignificance of an object and imply a more powerful and predominant force behind the camera, which is similar to the positions of the players who hold the power and controller behind the screen.

Eye-level angle is the most common view that people are used to seeing in everyday life. With different game mechanics, genres, and gameplay, the need for a variety of visual presentations will arise. An eye-level angle creates a fairly neutral impact and dispassionate space so players can concentrate more on the actions they make in games. Hence, most action games, fighting games, and battle themes in role-playing games are presented at the eye-level angle. In famous game series, such as Street Fighter and Mortal Kombat, the need for space is simply a platform where game characters can have fair competition. Even though the technology of computer graphics is advancing rapidly toward more gorgeous and complex content, the eye-level angle displays an impartial view of the match and adequately shows the physical actions.

The low angle, which viewed from below the eye level of the subject, makes the subject appear dominant, impressive, or in charge. There is not much gameplay viewing from a low angle-only a few shots from narrative cut scenes, or when encountering giant enemies or getting into steep surroundings. These situations only take place when it is required to look upward at specific objects or events temporarily, not throughout the entire gameplay. From a visual psychology point of view, in the low-angle view, the primary subject is amplified for its details and importance while the relatively more important information about the surrounding circumstances are weakened. That is, there are more functional considerations regarding the actual gameplay design of the viewing angles.

On the other hand, viewpoint fields can be discussed in two aspects: the field of view and the depth of field. Each represents the breadth and focus range of eye space. In fact, nowadays players can alter the two values according to their habits or the hardware performance in most games. The field of view, or the extent of the observable area, determines what players can see in games; in other words, it defines the boundaries of eye space. Within this field, players communicate with the primary subject with reference to the information they can get as a result of the distance to the subjected conditions of the viewing area, which decides how much information the players can acquire. In addition, it has a close 
correlation with viewing angles, especially the high-angle view. In the top-down style, strategy games usually have a wide field of view in order to observe a wide contact area so players can gain control over territories by thoroughly recognizing the circumstances they are in; accordingly, the visual details of each object may be reduced or simplified. Role-playing games, on the other hand, often display a closer perspective with a limited field of view. Players can only see the character and a certain area of the surroundings, yet physical actions and reactions can be made immediately for players when exploring in games with a more immersive approach. Therefore, the field of view has more to do with gameplay than graphics.

Regarding the depth of field, the view distance-in the same measure-determines how far into the distance players can see in games. Because depth perception is the visual ability of the human eye to perceive the 3D world, game design, likewise, attempts to represent $3 \mathrm{D}$ depth within the $2 \mathrm{D}$ plane in a variety of ways. Wolf (2008) has discussed the Z-axis development in video games from a historical viewpoint and mapped out several strategies. He asserts that creating a sense of depth will "fill the player's viewpoint with a larger and more detailed world of interconnected locations, encouraging involvement and giving players a virtual space to enter into where their attention is held and contained". Therefore, the depth of field in games richens the visual sense and provides depth clues for players during gameplay.

The interactive relations between game space and eye space should also be considered. Deciding which viewpoint to use and how to optimize it is a major topic. Viewpoint control is an essential part of the gameplay experience as well as the main issue between eye space and game space. In online gaming discussion forums, it is often found that players complain about problems caused by the in-game camera for reasons like it got stuck in the wall, the character disappears in the frame, the camera controls are too complicated, or it is causing dizziness and nausea. Those situations absolutely diminish the pleasure and interrupt the immersive playing experience. Attention, interaction, and comfort are the fundamental elements of an adaptive scrolling camera (Keren, 2015) and can be applied to the design of eye space as well. "Camera placement in games is usually directly controlled by the player or statically pre-defined by designers. Direct control of the camera by the player increases the complexity of the interaction and reduces the designer's control on game storytelling. A completely designer-driven camera releases the player from the burden of controlling the point of view, but might generate undesired camera behaviors." (Burelli, 2012) There are roughly two types of interactions between eye space and game space; game content and goals decide the suitable approaches with which to present the gameplay.

When reviewing articles related to viewpoint control, the more systematic discussions and organizations are mostly regarding computer graphics and concern virtual camera systems. For example, the four metaphors in viewpoint manipulation-eyeball in hand, world in hand, flying vehicle, and walking meta- 
phor (Christie, Olivier, \& Normand, 2008) - are based on the results of several studies (Ware \& Osborne, 1990). Analysis regarding the game camera is mainly made by Nitsche (2008) - he identifies five dominant camera behaviors in 3D games that are used within gameplay: following camera, overhead view, first-person point of view, predefined viewing frames and free camera. Based on his results, Colistra (2013) added the 2D legacy side-scrolling camera. In this paper, a different approach is proposed based on eye space. We place more stress on the importance of the participatory player and lessen the emphasis on the virtual camera system or issues of dimensionality, hoping to meet the actual playing experience of players and fit the variety of games. The analysis has been divided into two forms: fixed eye space and dynamic eye space.

Fixed eye space is non-controllable for players. It comprises three types: static, automatic, and predefined. Static means the eye space is constant and the information players need to know can be presented within one single frame. It can be found mostly in the games' early stages or with rather simpler mechanics. Some games might not seem static because they have elements such as moving lines, changing shapes, or even shifting backgrounds; however, the eye space is still unchanged. Static eye space provides a less disturbing viewpoint. Automatic eye space is made with programming and uses scripts or compiled languages to automatically control the movement of eye space. A common example is the following viewpoint, which tracks the main subjects and players who do not have the direct power to manipulate it. Most of the time, they can indirectly change the viewpoint of their controllable main subject. As a result, this relies upon the efficient and robust program design. Static and following viewpoints "play a large role in establishing a focal point and visually separating regions" (Milam, El-Nasr, Moura, \& Bartram, 2011). Predefined eye space is more concerned with the editing and presentation of the atmosphere from a functional aspect, such as changing different views not only for smooth gameplay but also for an expressive narrative.

On the other hand, dynamic eye space is adjustable and controllable for players, which means that players might have more involvement in the game space as well as predominance and freedom. At the same time, it might cause the focus to scatter, increase the complexity of control, cause more difficulty playing, and produce a longer gameplay adjustment time. To decrease the problems it might cause, some games implement supporting measures such as game tutorials, instruction pages, or the flexibility of customizing control settings. Dynamic viewpoint is dominated by two types: those that move along a single plane and those that rotate circularly around a central axis. The former allows players to pan and zoom in and out of the game space to observe certain areas or objects; it is better with broad space or numerous objects that cannot be displayed all at once. Visually, it is flat and distancing, as if looking through a microscope. The latter has a rotating center and is usually the main subject. The players can spin around to check their surroundings as though they are in the game space; the action of spinning the eye space is a way of creating a sense of perspective and immersion. 


\section{Conclusion and Future Work}

In this article, an original analytical framework of eye space is proposed with special attention placed on the significant interrelationships between players and game worlds. As a part of the human-computer interaction in a broad sense, eye space is the place where players and games meet and interact. In a way, it presents core game mechanics, reveals various game spaces, and shapes the players' experiences. While level design can be defined as "the container for gameplay" (Byrne, 2005), eye space, likewise, is the threshold for gameplay. A well-designed eye space should bring out the richness of visual content and meet the needs for functional gameplay, and more importantly, specify the standpoint in games for players. The discussions and results in this paper could be extended to other studies and, hopefully, be applicable to game design and development. Two major routes may be followed in future works. The first is to demonstrate and verify the framework with practical case studies and further discussions on eye space. The second is to delve into the relationships between game genres and different design strategies of eye space on players' experiences for gameplay.

"Video games vary greatly in terms of their construction and presentation, and video games need to be analyzed on all levels so that a critical vocabulary and analytical method can be developed as a starting point for the exploration of video games." (Taylor, 2002) Eye space, in its nature, also has a wide range of forms with the ability to sculpt different appearances of a game, which makes the analysis very hard to perform. In this study, the most fundamental elements and general situations are considered for building the framework in order to apply it to most of the games. The three aspects of this framework are closely related to each other, and each aspect has its own different qualities and defining elements. However, in order to provide a visually fluent gaming experience, they all have to work in combination. With the progress of a variety of technologies and creativities, the game has been a continual renewing medium. AR-for example, recently emerging technology-generates a whole new level of composite eye space by combining the real and virtual worlds, which creates a very different player experience. Further research should be done to contribute to this area. The relationship between players and games is very delicate and contains many possible factors. What is the unique language of presenting a game, and how does it work to keep players immersed? This research seeks to take the first step in this direction.

\section{Acknowledgements}

The authors would like to thank the committee members for their insightful remarks and providing enlightenment and critique on an earlier version of this manuscript. We are also very grateful to the editor and two anonymous reviewers for their valuable comments and constructive suggestions for improving the paper.

\section{References}

Adams, E. (2009). Fundamentals of Game Design (2 ed.). Berkeley, CA: New Riders. 
Bacher, D. (2008). Design Patterns in Level Design: Common Practices in Simulated Environment Construction. Ames, Iowa: Iowa State University.

Bayliss, P. (2007). Beings in the Game-World: Characters, Avatars, and Players. The IE'07 Proceedings of the 4th Australasian Conference on Interactive Entertainment, Australia.

Bowman, B., Elmqvist, N., \& Jankun-Kelly, T. J. (2012). Toward Visualization for Games: Theory, Design Space, and Patterns. IEEE Transactions on Visualization and Computer Graphics, 18, 1956-1968. https://doi.org/10.1109/TVCG.2012.77

Brathwaite, B., \& Schreiber, I. (2008). Challenges for Game Designers. USA: Cengage Learning.

Burelli, P. (2012). Interactive Virtual Cinematography. Copenhagen: IT University of Copenhagen.

Burelli, P. (2013). Virtual Cinematography in Games -Investigating the Impact on Player Experience. The Foundations of Digital Games.

Byrne, E. (2005). Game Level Design. USA: Charles River Media, Inc.

Christie, M., Olivier, P., \& Normand, J.-M. (2008). Camera Control in Computer Graphics. Computer Graphics Forum, 27, 2197-2218. https://doi.org/10.1111/j.1467-8659.2008.01181.x

Colistra, D. (2013). Video Games and Urban Visions. The NUL-New Urban Languages Conference Proceedings, Milan.

Emrich, A. (1993). MicroProse's Strategic Space Opera Is Rated XXXX. Computer Gaming World, September, 92-93.

Fagerholt, E., \& Lorentzon, M. (2009). Beyond the HUD-User Interfaces for Increased Player Immersion in FPS Games. Chalmers University of Technology, Göteborg.

Flynn, B. (2004). Games as Inhabited Spaces. Media International Australia, Incorporating Culture \& Policy, 110, 52-61.

Gazzard, A. (2013). Mazes in Videogames: Meaning, Metaphor and Design: McFarland.

Gosling, V. K., \& Crawford, G. (2011). Game Scenes: Theorizing Digital Game Audiences. Games and Culture, 6, 135-154. https://doi.org/10.1177/1555412010364979

Keren, I. (2015) Scroll Back-The Theory and Practice of Cameras in Side-Scrollers. Talk in Independent Games Summit: Game Developers Conference (GDC).

Klevjer, R. (2012). Enter the Avatar: The Phenomenology of Prosthetic Telepresence in Computer Games. In J. R. Sageng, H. Fossheim, \& T. M. Larsen (Eds.), The Philosophy of Computer Games (pp. 17-38). London \& New York, NY: Springer.

https://doi.org/10.1007/978-94-007-4249-9_3

Kneafsey, J. (2006). Virtual Cinematography for Computer Games. Dublin: Institute of Technology Blanchardstown.

Lankes, M., Mirlacher, T., Wagner, S., \& Hochleitner, W. (2014). Whom Are You Looking For? The Effects of Different Player Representation Relations on the Presence in Gaze-Based Games. In L. E. Nacke, \& T. C. Nicholas Graham (Eds.), Proceedings of the First ACM SIGCHI Annual Symposium on Computer-Human Interaction in Play (pp. 171-179). New York, NY: ACM.

Lin, T.-C., Shih, Z.-C., \& Tsai, Y.-T. (2004). Cinematic Camera Control in 3D Computer Games. The WSCG'2004: Short Communications: the 12-th International Conference in Central Europe on Computer Graphics, Visualization and Computer Vision.

Linderoth, J. (2005). Animated Game Pieces. Avatars as Roles, Tools and Props. Paper Presented at the Aesthetics of Play. http://www.aestheticsofplay.org/linderoth.php 
Logas, H., \& Muller, D. (2005). Mise-en-scène Applied to Level Design: Adapting a Holistic Approach to Level Design. The Proceedings of the 2005 DiGRA International Conference: Changing Views: Worlds in Play.

Manovich, L. (2001). The Language of New Media. Cambridge, MA: MIT Press.

Meldgaard, B. L. (2008). Perception, Action and Game Space. In S. Günzel, M. Liebe, \& D. Mersch (Eds.), The Conference Proceedings of the Philosophy of Computer Games 2008 (pp. 156-169). Potsdam: University Press.

Milam, D., El-Nasr, M. S., Moura, D., \& Bartram, L. (2011). Effect of Camera and Object Motion on Visual Load in 3D Games. In J. C. Anacleto, S. Fels, N. Graham, B. Kapralos, M. S. El-Nasr, \& K. Stanley (Eds.), The Proceedings of the 10th International Conference on Entertainment Computing (pp. 113-123). Berlin Heidelberg: Springer.

https://doi.org/10.1007/978-3-642-24500-8_12

Nitsche, M. (2005). Games, Montage, and the First Person Point of View. Paper presented at the DiGRA.

Nitsche, M. (2008). Video Game Spaces: Image, Play, and Structure in 3D Worlds. Cambridge, MA: MIT Press. https://doi.org/10.7551/mitpress/9780262141017.001.0001

Norman, D. A. (2002). The Design of Everyday Things (Reprint, Originally Published as The Psychology of Everyday Things, 1988 ed.). New York, NY: Basic Books.

Rehak, B. (2003). Playing at Being: Psychoanalysis and the Avatar. In M. J. P. Wolf, \& B. Perron (Eds.), The Video Game Theory Reader (pp. 103-128). New York, NY: Routledge.

Rollings, A., \& Morris, D. (2003). Game Architecture and Design: A New Edition. USA: New Riders.

Schell, J. (2008). The Art of Game Design: A Book of Lenses. Boca Raton, FL: CRC Press.

Taylor, L. N. (2002). Video Games: Perspective, Point-of-View, and Immersion. Gainesville, FL: University of Florida.

Thon, J. N. (2009). Perspective in Contemporary Computer Games. In P. Hühn, W. Schmid, \& J. Schönert (Eds.), Point of View, Perspective, and Focalization: Modeling Mediation in Narrative (pp. 279-299). Berlin: Walter de Gruyter.

Turkle, S. (2005, First Published in 1984). The Second Self: Computers and the Human Spirit (Twentieth Anniversary ed.). Cambridge, MA: The MIT Press.

Walz, S. P. (2010). Toward a Ludic Architecture: The Space of Play and Games. Pittsburgh, PA: ETC Press.

Ward, P. (2002). Picture Composition (2nd ed.). Waltham, MA: Focal Press.

Ware, C., \& Osborne, S. (1990). Exploration and Virtual Camera Control in Virtual Three Dimensional Environments. In M. J. Zyda (Ed.), I3D '90 Proceedings of the 1990 Symposium on Interactive 3D Graphics (pp. 175-183). New York, NY: ACM. https://doi.org/10.1145/91385.91442

Wolf, M. J. P. (2001). Space in the Video Game. In M. J. P. Wolf (Ed.), The Medium of the Video Game (pp. 51-75). Austin, TX: University of Texas Press.

Wolf, M. J. P. (2008). Z-Axis Development in the Video Game. In B. Perron, \& M. J. P. Wolf. (Eds.), The Video Game Theory Reader 2 (pp. 151-168). New York, NY: Routledge.

Wood, A. (2012). Recursive Space: Play and Creating Space. Games and Culture, 7, 87-105. https://doi.org/10.1177/1555412012440310 
Submit or recommend next manuscript to SCIRP and we will provide best service for you:

Accepting pre-submission inquiries through Email, Facebook, LinkedIn, Twitter, etc. A wide selection of journals (inclusive of 9 subjects, more than 200 journals)

Providing 24-hour high-quality service

User-friendly online submission system

Fair and swift peer-review system

Efficient typesetting and proofreading procedure

Display of the result of downloads and visits, as well as the number of cited articles Maximum dissemination of your research work

Submit your manuscript at: http://papersubmission.scirp.org/

Or contact adr@scirp.org 